\title{
Assessment of Morphological Variation in Irish Brassica oleracea Species
}

\author{
Mohamed A. El-Esawi ${ }^{1}$, Paula Bourke ${ }^{1}$, Kieran Germaine $^{2}$ \& Renee Malone ${ }^{1}$ \\ ${ }^{1}$ School of Food Science and Environmental Health, College of Sciences, Dublin Institute of Technology (DIT), \\ Dublin 1, Ireland \\ ${ }^{2}$ Department of Science and Health, Institute of Technology Carlow, Carlow, Ireland \\ Correspondence: Mohamed A. El-Esawi, School of Food Science and Environmental Health, Dublin Institute of \\ Technology (DIT), Dublin 1, Ireland. Permanent address: Botany Department, Faculty of Science, Tanta \\ University, Tanta, Egypt. E-mail: elesawi2005@yahoo.com.
}

Received: May 2, 2012 Accepted: May 17, 2012 Online Published: August 24, 2012

doi:10.5539/jas.v4n10p20 URL: http://dx.doi.org/10.5539/jas.v4n10p20

\begin{abstract}
This study aimed at assessing the morphological variation in 25 accessions of an Irish collection of Brassica oleracea using 44 morphological traits. The morphological traits showed a pronounced variation among accessions. Vegetative preflowering and maturity traits revealed higher variation than seedling traits. Of the 44 morphological descriptors used, 28 were significantly different and proved useful in examining diversity and differentiating among accessions studied. Principal component analysis (PCA) showed variation among accessions, where $87.24 \%$ of the total variation in the morphological data was separated on the first five components, indicating a high degree of correlation among traits studied. Cluster analysis grouped the 25 accessions into 4 main groups, corresponding to kales, cauliflowers, cabbages and sprouts, and also showed a sort of correlation between clustering pattern and eco-geographical distribution of the accessions. Furthermore, this study identified 9 highly diverse accessions, providing opportunities for optimising parental sources in future breeding programs to develop new or more productive Brassica varieties. Morphological traits were deemed useful for assessing the diversity and relationships in Irish Brassica oleracea species.
\end{abstract}

Keywords: Brassica oleracea, morphological variation, relationships, trait

\section{Introduction}

The genus Brassica L. is one of the most economically important genera in the tribe Brassiceae, which in turn belongs to the family Brassicaceae (Rakow, 2004). This genus comprises a diverse group of species including major vegetable and oilseed crops with a wide range of agronomic traits (Rich, 1991; Christopher et al., 2005). It contains six economically important species with great genetic and morphological diversity which are cultivated worldwide (Saha et al., 2008). Three of these species are diploid (Brassica oleracea, $2 \mathrm{n}=18 ;$ Brassica rapa, $2 \mathrm{n}=$ 20; Brassica nigra, $2 \mathrm{n}=16$ ), and three are amphidiploid (Brassica napus, $2 \mathrm{n}=38 ;$ Brassica juncea, $2 \mathrm{n}=36$; Brassica carinata, $2 \mathrm{n}=34$ ). Brassica species play an important role in agriculture and horticulture, as well as contributing both to the economy and health of populations around the world (King, 2005; Zhao, 2007).

Brassica oleracea L. is an important vegetable crop species which includes many vegetable cultivars called cole crops (Katz, 2003). These cole crops comprise cabbage (Brassica oleracea subspecies capitata), cauliflower (Brassica oleracea subspecies botrytis), brussels sprout (Brassica oleracea subspecies gemmifera), broccoli (Brassica oleracea subspecies italica), Kale and collards (Brassica oleracea subspecies acephala), and kohlrabi (Brassica oleracea subspecies gongylodes). Cole crops have extreme morphological characteristics. Examples of such morphologies include the enlarged infloresences of cauliflower and broccoli; the enlarged stems of kohlrabi and marrowstem kale; the enlarged single apical bud of cabbage; and the several axillary buds of brussels sprout (Paterson et al., 2001; Hong et al., 2008).

Assessment of diversity is an essential component in germplasm characterisation and conservation, which in turn are essential to the continued maintenance and improvement of agricultural production and, thus, to sustainable development and poverty alleviation (Karp et al., 1997). Morphological variation in plant species has been described for traits controlled by a single or multiple gene systems. However, the variation patterns in the morphological traits are considered to be the result of both genetic and environmental attributes (Rohlf et al., 1990; Rohlf, 2000). Morphological traits have been used to assess the genetic variation and relationships among populations of different plant species, for example, mungbean (Arshad et al., 2009; Tantasawat et al., 2010), 
soybean (Iqbal et al., 2008), rice (Bibi et al., 2009), eggplant (Tümbilen et al., 2011) and Brassica (Rabbani et al., 1999; Kop et al., 2003; Balkaya et al., 2005).

Rabbani et al. (1999) used the morphological traits to assess the variation in oilseed mustard. 35 phenotypic traits were recorded for all the 52 accessions studied from seedling emergence up to crop harvest. The results revealed a considerable level of diversity among all tested accessions for various traits. Seedling traits showed less variation, while the largest variation was found for flowering and maturity stage traits. Generally, a low correlation was observed among different traits, however, some of the related characters were significantly correlated with each other. The results also indicated that oilseed mustard in Pakistan has narrow genetic base and experiences a high level of genetic erosion.

Balkaya et al. (2005) determined the differences regarding morphological variation of white head cabbage (Brassica oleracea var. capitata subvar. alba) genetic resources collected from different regions of Turkey. Their cluster analysis based on 12 quantitative and 10 qualitative variables identified 10 groups. Morphological variability was high among the white head cabbage genotypes of Turkey. The genotypes also displayed a wide diversity of characters for selection and combination of interesting genotypes for important characters to obtain improved varieties.

Although significant progress has been made in the past decade, Brassica biodiversity loss has not been halted in the island of Ireland. The status of many of Irish habitats and species is judged to be poor (NBP, 2011). Moreover, information on the genetic diversity and relationships in Irish Brassica oleracea species is currently very limited. Therefore, the main aim of this study was to assess the morphological variation and character association in Irish Brassica oleracea accessions based on morphological traits, thus providing a correct taxonomic ranging and a precise characterisation of these accessions.

\section{Materials and Methods}

\subsection{Plant Material}

Twenty-Five accessions of Irish Brasssica oleracea were obtained from the germplasm collection maintained at the Horticultural Research Institute (HRI), Wellsbourne, United Kingdom (Table 1). These accessions were chosen based on their sampling site covering a broad and diverse geographic range of the island of Ireland. However, the selected accessions represented 4 subspecies within Brassica oleracea species (Brassica oleracea capitata, Brassica oleracea acephala, Brassica oleracea botrytis and Brassica oleracea gemmifera).

Table 1. Accession numbers, crop names, and collection sites of the accessions of Brassica oleracea studied

\begin{tabular}{|c|c|c|c|c|c|}
\hline No. & Accession Number & Subspecies & Accession Name & Crop name & Collection site \\
\hline 1 & HRIGRU 4502 & Brassica oleracea acephala & Marrow Stem & fodder kale & Kildare \\
\hline 2 & HRIGRU 4503 & Brassica oleracea acephala & Thousand Head & fodder kale & Kildare \\
\hline 3 & HRIGRU 7229 & Brassica oleracea acephala & Cut and Come Again & kale & Tipperary \\
\hline 4 & HRIGRU 7556 & Brassica oleracea acephala & Cut and Come Again & kale & Cork \\
\hline 5 & HRIGRU 7227 & Brassica oleracea acephala & Raggedy Jack & kale & Sligo \\
\hline 6 & HRIGRU 4492 & Brassica oleracea botrytis & Winter Roscoff & winter cauliflower & Dublin \\
\hline 7 & HRIGRU 4565 & Brassica oleracea botrytis & & winter cauliflower & Cork \\
\hline 8 & HRIGRU 4495 & Brassica oleracea botrytis & Winter Roscoff & winter cauliflower & Ballykea \\
\hline 9 & HRIGRU 4579 & Brassica oleracea capitata & Flat Dutch & cattle cabbage & Donegal \\
\hline 10 & HRIGRU 4561 & Brassica oleracea capitata & Flat Dutch & cattle cabbage & Galway \\
\hline 11 & HRIGRU 4508 & Brassica oleracea capitata & Flat Dutch & cattle cabbage & Ballina \\
\hline 12 & HRIGRU 4506 & Brassica oleracea capitata & Flat Dutch & cattle cabbage & Ballinrobe \\
\hline 13 & HRIGRU 4585 & Brassica oleracea capitata & Flat Dutch & common cabbage & Donegal \\
\hline 14 & HRIGRU 4586 & Brassica oleracea capitata & Flat Dutch & common cabbage & Mayo \\
\hline 15 & HRIGRU 4497 & Brassica oleracea capitata & Flat Dutch & cabbage & Roscommon \\
\hline 16 & HRIGRU 4498 & Brassica oleracea capitata & Flat Dutch & cabbage & Roscommon \\
\hline 17 & HRIGRU 4588 & Brassica oleracea capitata & Flat Dutch & cabbage & Donegal \\
\hline 18 & HRIGRU 5915 & Brassica oleracea capitata & Flat Dutch & cabbage & Limerick \\
\hline 19 & HRIGRU12532 & Brassica oleracea capitata & Delaway Cabbage & cabbage & Mayo \\
\hline 20 & HRIGRU 4566 & Brassica oleracea capitata & & spring cabbage & Cork \\
\hline 21 & HRIGRU 4564 & Brassica oleracea capitata & & spring cabbage & Cork \\
\hline 22 & HRIGRU 4571 & Brassica oleracea capitata & & spring cabbage & Cork \\
\hline 23 & HRIGRU 5914 & Brassica oleracea capitata & Spring Greens & spring cabbage & Limerick \\
\hline 24 & HRIGRU 4491 & Brassica oleracea gemmifera & & brussels sprout & Dublin \\
\hline 25 & HRIGRU 4494 & Brassica oleracea gemmifera & & brussels sprout & Dublin \\
\hline
\end{tabular}




\subsection{Experimental Design and Plant Growth}

Seeds were sown in PPE modules of $12 \times 12$ cells filled with seedling compost (Shamrock) in the greenhouse facilities at the Teagasc Horticultural Research centre in Kinsealy, Dublin, Ireland. Four weeks after germination, the seedlings were transplanted to larger PPE pots containing compost in the greenhouse. After a further 5 weeks, the modules were removed and placed on a bench outside the greenhouse in order to harden the seedlings off for 2 weeks. Land plots were prepared within the field trial site at Teagasc Kinsealy. Prior to transplanting, the seedlings were treated with Dursan (an insecticide for the control of caterpillars, cabbage root fly and cutworms). The seedlings were then transferred into 5 blocks in the field, with 5 accessions per block in a randomized scheme (Figure 1). Sowing and growing the accessions at different time points are shown in Figure 2.

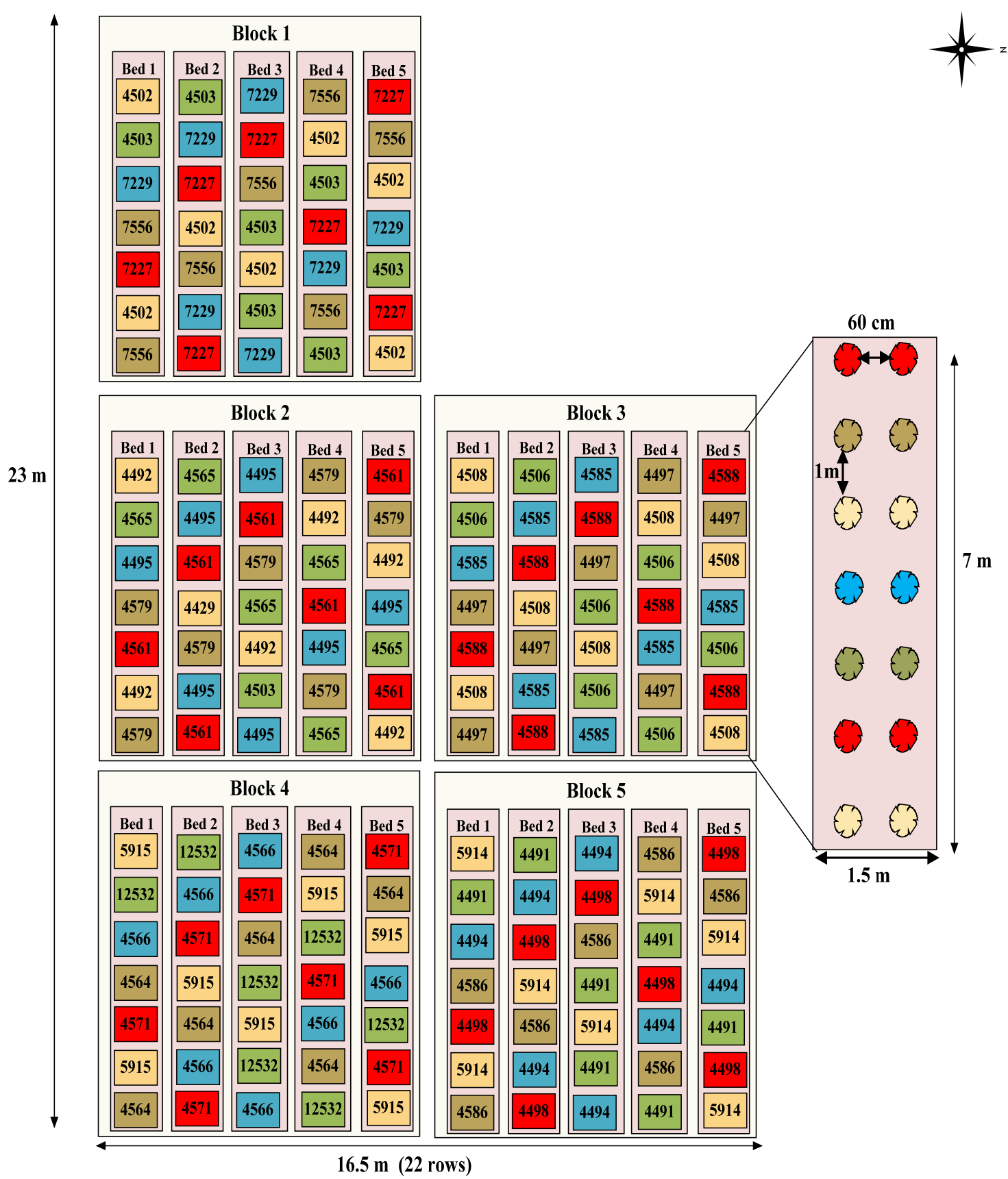

2 plants per plot: 7 plots per accession $=14$ plants in total per accession

Figure 1. Randomised planting scheme used for transplanting Brassica oleracea accessions (the numbers in the blocks refers to the accessions numbers) 


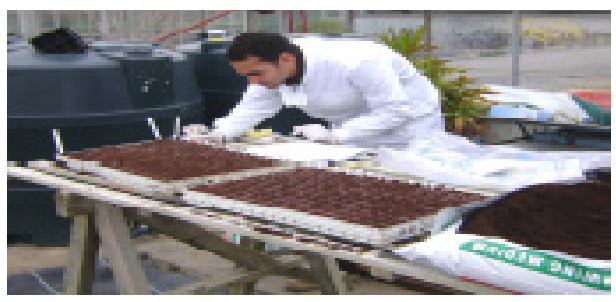

$\mathbf{a}$

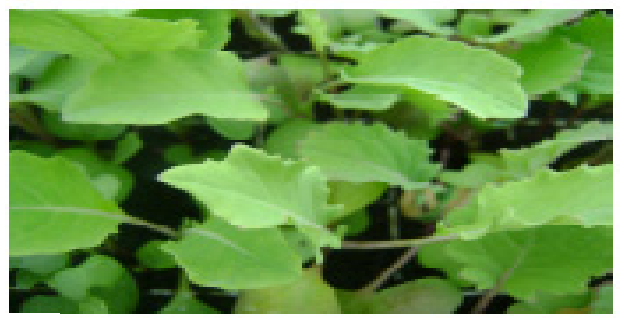

c

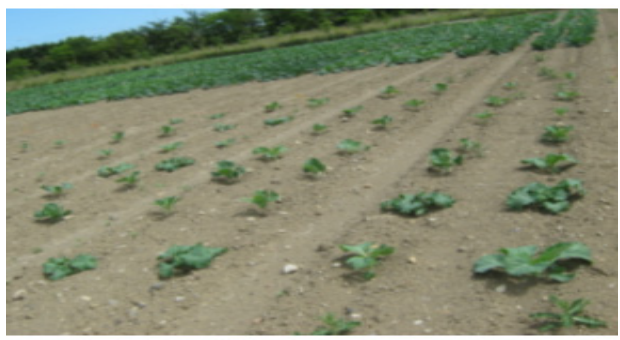

e
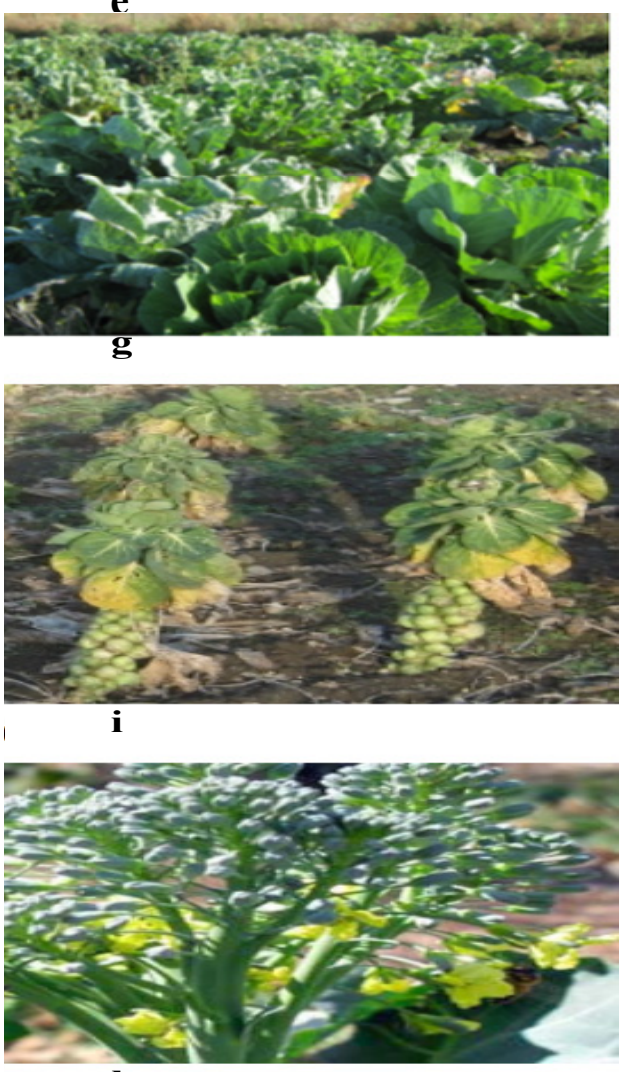

$\mathbf{k}$

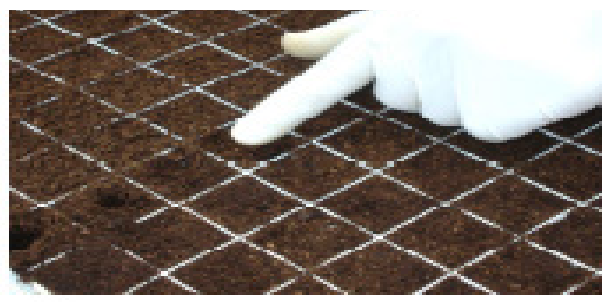

h

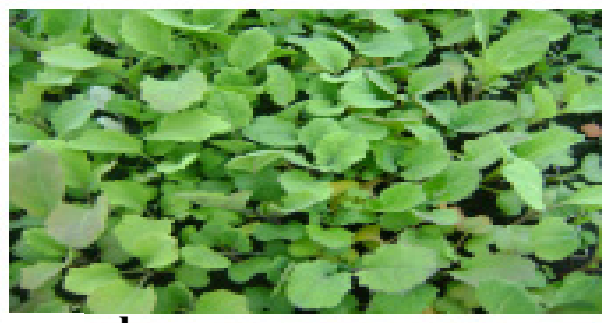

d

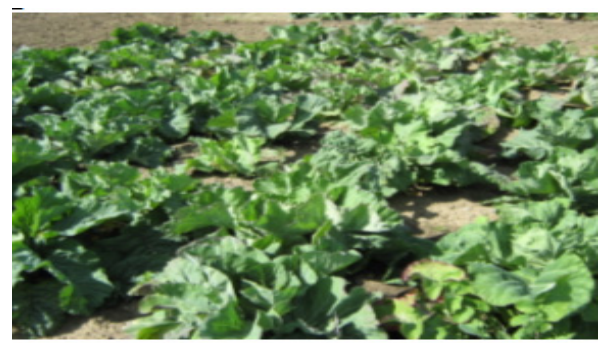

f
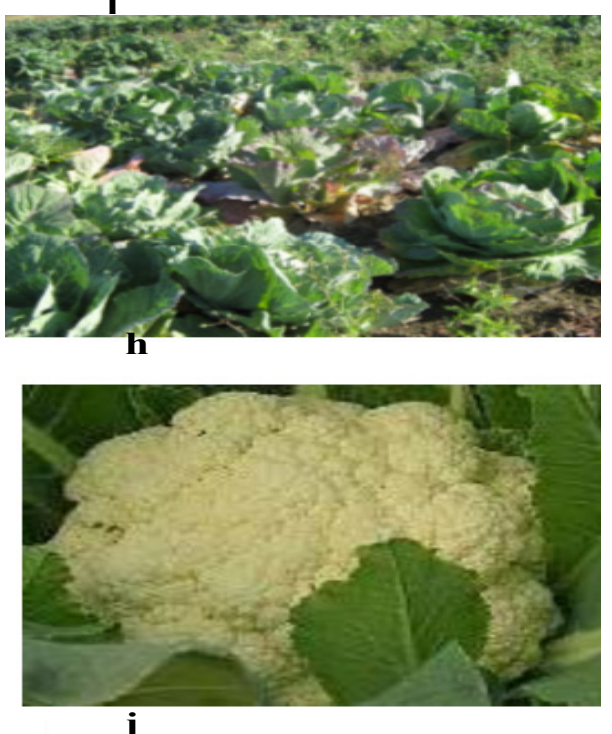

$\mathbf{j}$

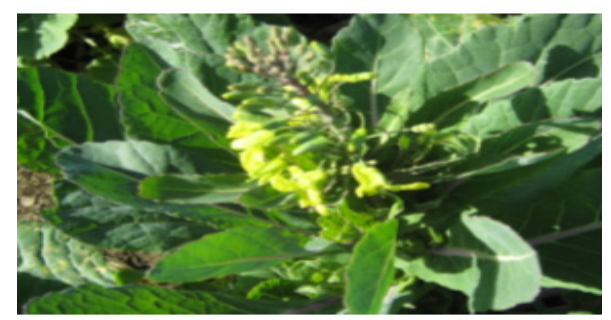

Figure 2. Sowing Brassica accessions at Teagasc Kinsealy (a, b, March 2009), seedling stage (c, d, April 2009), transplanting into field site (e, June 2009), growth of the plants (f, August 2009; $\mathbf{g}$, h, September 2009), mature pre-flowering stage (i, j, October 2009), and flowering stage (k, l, December 2009) 


\subsection{Evaluation of Morphological Traits}

Forty-four discriminating descriptors were selected from the list of morphological descriptors for Brassica and Raphanus published by the International Board for Plant Genetic Resources (IBPGR, 1990). These descriptors were considered to be suitable for Brassica oleracea, and were used in this study to characterize 10 plants of each of the 25 accessions studied. The descriptors selected included 5 descriptors at the seedling stage, 32 descriptors at the mature pre-flowering stage and 7 descriptors at the flowering stage (Table 2).

Table 2. Morphological traits and descriptor states applied to all accessions of Brassica oleracea studied (IBPGR, 1990)

\begin{tabular}{|c|c|c|}
\hline $\begin{array}{l}\text { IBPGR } \\
\text { Descriptor } \\
\text { No. }\end{array}$ & Trait & Descriptor state \\
\hline \multicolumn{3}{|l|}{ 4.1 Seedling } \\
\hline \multirow[t]{2}{*}{ 4.1.1 } & Hypocotyl colour & Observe at 5-leaf stage \\
\hline & & $\begin{array}{l}\text { 1, White; 2, Pale green; 3, Green; 4, Pink; 5, Red; 6, Purple; 7, } \\
\text { Other }\end{array}$ \\
\hline 4.1 .2 & Seedling leaf colour & $\begin{array}{l}\text { 1, White green; 2, Yellow green; 3, Light green; 4, Green; 5, Dark } \\
\text { green; 6, Purple green; 7, Purple; 8, Other }\end{array}$ \\
\hline 4.1 .3 & Seedling leaf (marginal incisions) & $\begin{array}{l}0 \text {, Entire; } 1 \text {, Crenate; } 2 \text {, Dentate; } 3 \text {, Serrate; } 4 \text {, Undulate; } 5 \text {, Doubly } \\
\text { dentate; } 6 \text {, Other }\end{array}$ \\
\hline 4.1.4 & Seedling pubesence & 0, Glabrous; 1 , Very sparse; 3 , Sparse; 5 , Intermediate; 7 , Abundant \\
\hline 4.1 .5 & Juvenile development & 3, Slow; 5 , Intermediate; 7 , Fast \\
\hline \multicolumn{3}{|c|}{ 4.2 Vegetative preflowering mature } \\
\hline \multirow[t]{2}{*}{ 4.2.2 } & Plant growth habit & $\begin{array}{l}\text { 1, Shortened nonbranching stem supporting leafy rosette; } 2 \text {, } \\
\text { Shortened nonbranching stem terminating in leafy head; } 3 \text {, } \\
\text { Extremely shortened branching stems forming leafy crown; 4, } \\
\text { Elongate and/or enlarged nonbranching stem (tubers) supporting } \\
\text { leaves and/or head; 5, Elongate nonbranching stem supporting } \\
\text { enlarged compact axillary buds; 6, Elongate branching stems } \\
\text { supporting leaves and/or heads; 7, Elongate nonbranching stem } \\
\text { terminating in enlarged floral or prefloral apex (curd); 8, Elongate } \\
\text { branching stems terminating in enlarged floral or prefloral apices; }\end{array}$ \\
\hline & & 9, Enlarged root; 10, Other \\
\hline 4.2 .9 & Lodging & 3, Low; 5, Intermediate; 7, High \\
\hline 4.2 .11 & Number of leaves (estimated) & 3, Few; 5, Intermediate; 7, Many \\
\hline \multirow[t]{2}{*}{4.2 .16} & Leaf blade shape in outline & $\begin{array}{l}\text { 1, Orbicular; 2, Elliptic; 3, Obovate; 4, Spathulate; 5, Ovate; } 6 \text {, } \\
\text { Lanceolate; }\end{array}$ \\
\hline & & 7, Oblong; 8, Other \\
\hline 4.2 .17 & Leaf division (margin) & $\begin{array}{l}0 \text {, Entire; } 1 \text {, Crenate; } 2 \text {, Dentate; } 3 \text {, Serrate; } 4 \text {, Undulate; } 5 \text {, Doubly } \\
\text { dentate; } 6 \text {, Other }\end{array}$ \\
\hline 4.2 .18 & Leaf division (incision) & 1, Entire; 2, Sinuate; 3, Lyrate; 4, Lancerate; 5, Other \\
\hline 4.2 .19 & Leaf apex shape & $\begin{array}{l}\text { 2, Acute; } 4 \text {, Intermediate; } 6 \text {, Rounded; } \\
8 \text {, Broadly Rounded }\end{array}$ \\
\hline 4.2 .24 & Leaf colour & $\begin{array}{l}\text { 1, Yellow green; 2, Light green; 3, Green; 4, Dark green; 5, Purple } \\
\text { green; 6, Purple; 7, Other }\end{array}$ \\
\hline 4.2 .25 & Leaf hairiness & 0, Absent; 3, Sparse; 5, Intermediate; 7 , Abundant \\
\hline 4.2 .26 & Leaf bloom & 0, Absent, glossy; 3, Low; 5, Intermediate; 7, High, glaucous \\
\hline 4.2 .32 & Petiole section & 3, Round; 5, Semiround; 7, Flat \\
\hline 4.2 .33 & Petiole and/or midvein colour & 1, White; 2, Light green; 3, Green; 4, Purple; 5 Red; 6 Other \\
\hline 4.2 .34 & Heading habit & $\begin{array}{l}\text { Observe at harvest, head-forming types only } \\
0 \text {, Nonheading; } 5 \text {, Semiheading; } 7 \text {, Heading }\end{array}$ \\
\hline 4.2 .35 & Head shape in longitudinal section & $\begin{array}{l}\text { 1, Triangular; 2, Ovate; } 3 \text {, Obovate; } 4 \text {, Elliptic; } 5 \text {, Spheric; } 6 \text {, } \\
\text { Cylindric; } 7 \text {, Transverse elliptic; } 8 \text {, Other }\end{array}$ \\
\hline
\end{tabular}


4.3 Flowering plant characteristics
4.3.2
Days to flowering
4.3.7
Flower stalk colour

$\begin{array}{ll}\text { 4.3.10 } & \text { Flower stalk length, estimate } \\ \text { 4.3.11 } & \text { Flower stalk internode length } \\ 4.3 .15 & \text { Flowering plant hairiness } \\ 4.3 .16 & \text { Flower colour variability in the crop } \\ 4.3 .17 & \text { Petal colour }\end{array}$

1, Leaves curled outwards; 2, Erect; 3, Curled inwards; 4, Slight overlap; 5, Strong overlap

3, Exposed; 5, Intermediate; 7, Covered

1, Yellow green; 2, Pale green; 3, Green; 4, Dark green; 5, Red green; 6 , Red or purple

3, Small; 5, Intermediate; 7, Large

3, Low (soft, loose); 5, Intermediate; 7, High (firm, hard)

Measure median transverse section

Measured at widest point

3, Short; 5, Intermediate; 7, Long

1, White; 2, Yellow; 3, Pale green; 4, Green; 5, Red green; 6, Red or purple

3, Low; 5, Intermediate; 7, High

3, Low; 5, Intermediate; 7, High

3, Early; 5, Midseason; 7, Late

3, Low; 5, Intermediate; 7, High

3, Few; 5, Intermediate; 7, Many

3, Small; 5, Intermediate; 7, Large

3, Loose; 5, Intermediate; 7, Firm

1, Light green; 2, Green; 3, Dark green; 5 Red/Purple green; 6 Red/purple

1, Single flower raceme; 2 , Enlarged stem with terminally branched raceme; 3, Loosely branched small terminal heads; 4, Terminal head with smaller heads on axillary shoots; 5 , Compact head of regularly packed subheads; 6 , Single compact head of irregularly packed subheads; 7 , Other

Number of days from sowing to when $50 \%$ of plants first flower Observed at base of stem

1, White; 2, Yellow green; 3, Green; 4, Red green; 5, Red; 6, Purple; 7, Other

3, Short; 5, Intermediate; 7, Long

3, Short; 5, Intermediate; 7, Long

0, Absent; 3, Few hairs; 5, Intermediate; 7, Hairy

3, Uniform; 5, Intermediate; 7, Highly variable, many colours

1, White; 2, Pale yellow; 3, Yellow; 4, Orange yellow; 5, Pink; 6, Red; 7, Purple; 8, Other

\subsection{Data Analysis}

The morphological data were standardised and converted to z-scores to avoid bias in the analysis due to scale differences in the variables (Legendre and Legendre, 1998). The standardised dataset was then used to estimate the genetic diversity and relationships among the accessions which were evaluated by Jaccard similarity index, cluster analysis, factor analysis, analysis of variance and correlation coefficients. These multivariate analyses were made using the software package SYSTAT for Windows, version 7.0 copyright (C) 1997, SPSS INC. A dendrogram was constructed through the average linkage-joining rule.

\section{Results}

\subsection{Variation of Morphological Traits and Their Statistical Parameters}

Of the 44 morphological traits analyzed in all the 25 accessions studied, 42 were polymorphic and therefore further used for the diversity analysis (Table 3). However, the hypocotyl colour and seedling leaf (marginal incisions) traits had no taxonomic value among the 25 accessions studied. Many traits showed a high variability among the accessions studied, for example, petiole section, petiole and midvein colour, leaf colour, primary colour of outer head leaves, and leaf division (incision). The minimum, maximum, range, mean, standard deviation, percent and 
coefficient of variation were estimated for morphological traits analyzed in all the 25 accessions studied (Table 3 ). The quantitative characters measured exhibited broad variability. Head length, for example ranged from 0 to 22 $\mathrm{cm}$, head diameter ranged from 0 to $19.5 \mathrm{~cm}$, and days to flowering ranged from 240 to 307 . Standard deviation (SD) and coefficient of variance $(\mathrm{CV} \%)$ showed high values for most traits, e.g. head length $(\mathrm{SD}=8.457 ; \mathrm{CV}=$ $0.606)$ and head diameter $(\mathrm{SD}=7.541 ; \mathrm{CV}=0.612)$. The coefficient of variance $(\mathrm{CV} \%)$ showed a low value for days to flowering $(\mathrm{CV}=0.078)$.

Table 3. Statistical parameters for quantitative and qualitative traits of Brassica oleracea accessions (see Table 1 for identification of accessions numbers)

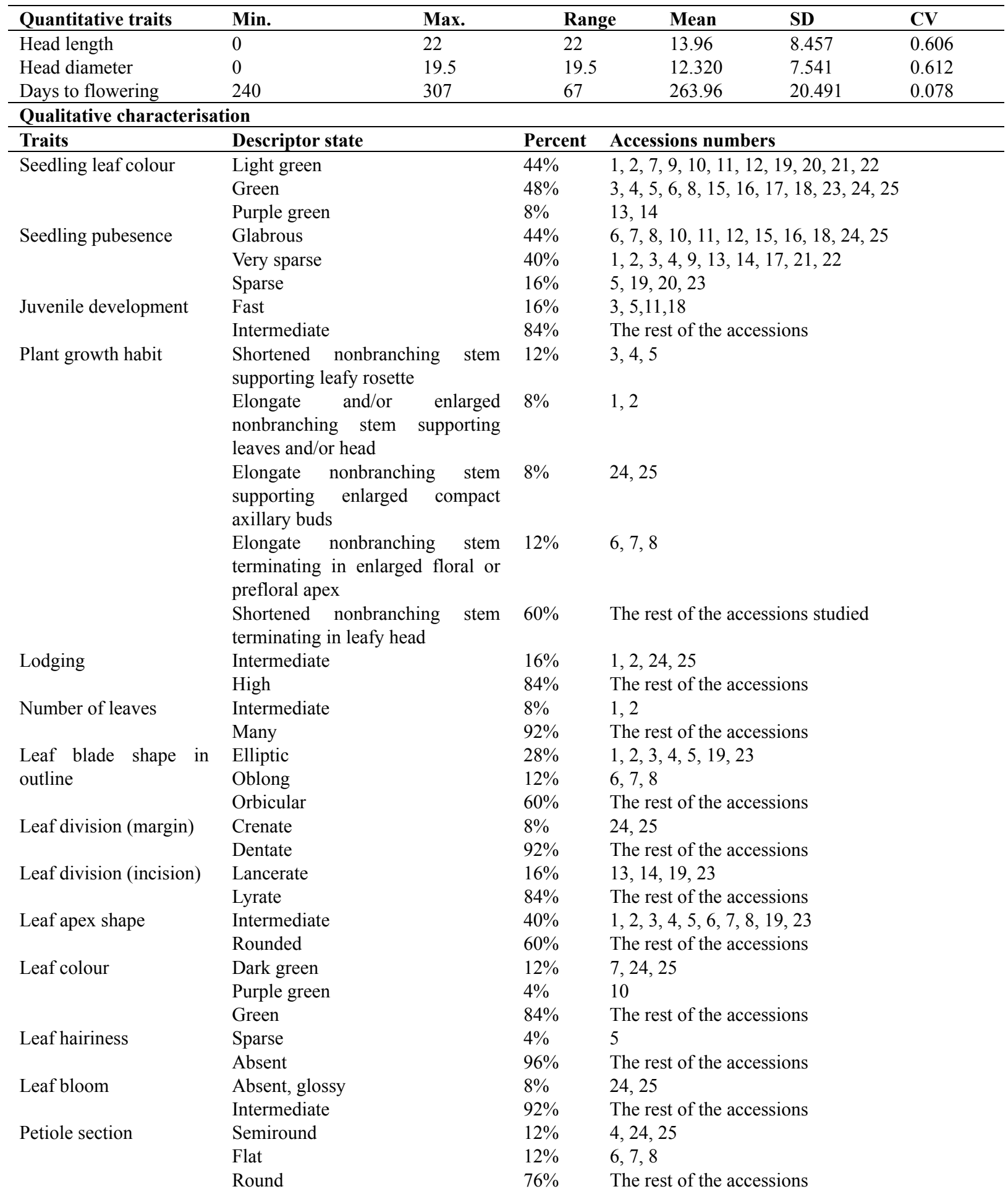




\begin{tabular}{|c|c|c|c|}
\hline \multirow{3}{*}{$\begin{array}{l}\text { Petiole and/or midvein } \\
\text { colour }\end{array}$} & Green & $8 \%$ & 24,25 \\
\hline & Purple & $4 \%$ & 19 \\
\hline & Light green & $88 \%$ & The rest of the accessions \\
\hline \multirow[t]{2}{*}{ Heading habit } & Nonheading & $20 \%$ & $1,2,3,4,5$ \\
\hline & Heading & $80 \%$ & The rest of the accessions \\
\hline \multirow{3}{*}{$\begin{array}{l}\text { Head shape in } \\
\text { longitudinal section } \\
\text { Head-forming leaf } \\
\text { overlap at terminal } \\
\text { region }\end{array}$} & Spheric & $80 \%$ & $\begin{array}{l}6,7,8,9,10,11,12,13,14,15,16,17,18 \\
19,20,21,22,23,24,25\end{array}$ \\
\hline & Curled inwards & $12 \%$ & $6,7,8$ \\
\hline & Strong overlap & $68 \%$ & $\begin{array}{l}9,10,11,12,13,14,15,16,17,18,19,20 \\
21,22,23,24,25\end{array}$ \\
\hline \multirow{5}{*}{$\begin{array}{l}\text { Head cover from } \\
\text { subtending leaves } \\
\text { Primary colour of outer } \\
\text { head leaves }\end{array}$} & Intermediate & $20 \%$ & $6,7,8,24,25$ \\
\hline & Covered & $60 \%$ & $9,10,11,12,13,14,15,16,17,18,19,20,21,22,23$ \\
\hline & Green & $64 \%$ & $6,8,9,10,12,13,14,15,16,17,18,19,20,21,22,23$ \\
\hline & Dark green & $12 \%$ & $7,24,25$ \\
\hline & Red green & $4 \%$ & 11 \\
\hline \multirow{3}{*}{$\begin{array}{l}\text { Head size in relation to } \\
\text { plant size }\end{array}$} & Small & $8 \%$ & 24,25 \\
\hline & Intermediate & $12 \%$ & $6,7,8$ \\
\hline & Large & $60 \%$ & $9,10,11,12,13,14,15,16,17,18,19,20,21,22,23$ \\
\hline Head solidity & High (firm, hard) & $80 \%$ & $\begin{array}{l}6,7,8,9,10,11,12,13,14,15,16,17,18 \\
19,20,21,22,23,24,25\end{array}$ \\
\hline \multirow{3}{*}{$\begin{array}{l}\text { Stem length in head, } \\
\text { estimate } \\
\text { Primary colour inside } \\
\text { cut }\end{array}$} & Intermediate & $80 \%$ & $\begin{array}{l}6,7,8,9,10,11,12,13,14,15,16,17,18 \\
19,20,21,22,23,24,25\end{array}$ \\
\hline & White & $52 \%$ & $6,7,8,12,15,16,17,18,19,20,21,22,23$ \\
\hline & Pale green & $28 \%$ & $9,10,11,13,14,24,25$ \\
\hline \multirow{2}{*}{$\begin{array}{l}\text { Tendency of head to } \\
\text { split }\end{array}$} & Low & $36 \%$ & $15,16,17,18,19,20,21,22,23$ \\
\hline & Intermediate & $44 \%$ & $6,7,8,9,10,11,12,13,14,24,25$ \\
\hline Head holding ability & High & $80 \%$ & $\begin{array}{l}6,7,8,9,10,11,12,13,14,15,16,17,18 \\
19,20,21,22,23,24,25\end{array}$ \\
\hline \multirow{5}{*}{$\begin{array}{l}\text { Relative time of } \\
\text { maturity } \\
\text { Leaf retention on stem }\end{array}$} & Late & $32 \%$ & $1,2,3,4,5,6,7,8$ \\
\hline & Midseason & $68 \%$ & The rest of the accessions \\
\hline & Low & $8 \%$ & 24,25 \\
\hline & Intermediate & $4 \%$ & 5 \\
\hline & High & $88 \%$ & The rest of the accessions \\
\hline \multirow{2}{*}{$\begin{array}{l}\text { Number of shoots or } \\
\text { enlarged buds } \\
\text { Size of enlarged } \\
\text { vegetative buds }\end{array}$} & Many & $8 \%$ & 24,25 \\
\hline & Large & $8 \%$ & 24,25 \\
\hline $\begin{array}{l}\text { Firmness of enlarged } \\
\text { vegetative buds }\end{array}$ & Firm & $8 \%$ & 24,25 \\
\hline \multirow{2}{*}{$\begin{array}{l}\text { Colour of enlarged } \\
\text { vegetative buds or } \\
\text { shoots }\end{array}$} & Green & $4 \%$ & 25 \\
\hline & Dark green & $4 \%$ & 24 \\
\hline $\begin{array}{l}\text { Floral apex branching } \\
\text { pattern }\end{array}$ & $\begin{array}{l}\text { Single compact head of irregularly } \\
\text { packed subheads }\end{array}$ & $12 \%$ & $6,7,8$ \\
\hline \multirow[t]{3}{*}{ Flower stalk colour } & Green & $12 \%$ & $8,24,25$ \\
\hline & Red green & $20 \%$ & $1,2,3,4,5$ \\
\hline & Yellow green & $68 \%$ & The rest of the accessions \\
\hline \multirow{3}{*}{$\begin{array}{l}\text { Flower stalk length, } \\
\text { estimate }\end{array}$} & Intermediate & $32 \%$ & $1,2,3,4,5,6,7,8$ \\
\hline & Long & $8 \%$ & 24,25 \\
\hline & Short & $60 \%$ & The rest of the accessions \\
\hline \multirow{3}{*}{$\begin{array}{l}\text { Flower stalk internode } \\
\text { length }\end{array}$} & Intermediate & $32 \%$ & $1,2,3,4,5,6,7,8$ \\
\hline & Long & $8 \%$ & 24,25 \\
\hline & Short & $60 \%$ & The rest of the accessions \\
\hline \multirow{6}{*}{$\begin{array}{l}\text { Flowering plant } \\
\text { hairiness } \\
\text { Flower colour } \\
\text { variability in the crop } \\
\text { Petal colour }\end{array}$} & Few hairs & $8 \%$ & 24,25 \\
\hline & Absent & $92 \%$ & The rest of the accessions \\
\hline & Intermediate & $4 \%$ & 25 \\
\hline & Uniform & $96 \%$ & The rest of the accessions \\
\hline & Yellow & $32 \%$ & $1,3,4,9,11,15,18,24$ \\
\hline & Pale yellow & $68 \%$ & The rest of the accessions \\
\hline
\end{tabular}


Most of accessions were of green seedling leaf color (48\%) (Table 3). Also light green color of seedling leaf is represented by a high percentage (30\%). While only the 2 accessions of common cabbage were of purple green seedling leaf color. Most of accessions were of glabrous seedling pubescence, intermediate juvenile development, shortened nonbranching stem terminating in leafy head, high lodging, many number of leaves, orbicular leaf blade shape, dentate leaf margin, lyrate leaf incision, rounded leaf apex, green leaf colour, absent leaf hairiness, intermediate leaf bloom, rounded petiole section, spheric head shape, strong head forming leaf overlap, covered head, green primary colour of outer head leaves, high head solidity, intermediate stem length in head, white primary colour inside cut, high holding ability, high leaf retention on stem, yellow green flower stalk, short flower stalk length, absent flowering plant hairiness and pale yellow petals (Table 3).

\subsection{Cluster Analysis}

Morphological data differentiated all the accessions of Brassica oleracea studied. The phenogram constructed using each accession as an OTU (operational taxonomic unit) was performed on the basis of the collected morphological data of the 25 accessions of Brassica oleracea (Figure 3). This phenogram exhibited that all the genetic distance among the examined accessions was around $65 \%$, and showed 4 major groups. The first group contained the 2 accessions of brussels sprout. The second group contained all accessions of kale and fodder kale. The third group was homogenous and contained all accessions of winter cauliflower. The fourth group split into 4 subgroups. Furthermore, the accessions of most cabbage types were distributed among different clusters within these subgroups. None of the studied cabbage types formed a distinct cluster except the 2 accessions of common cabbage which were separated in one cluster within the third subgroup. The accessions of cabbages HRIGRU5915 and HRIGRU4588 were very similar to the accessions of cabbages HRIGRU4497 and HRIGRU4498.

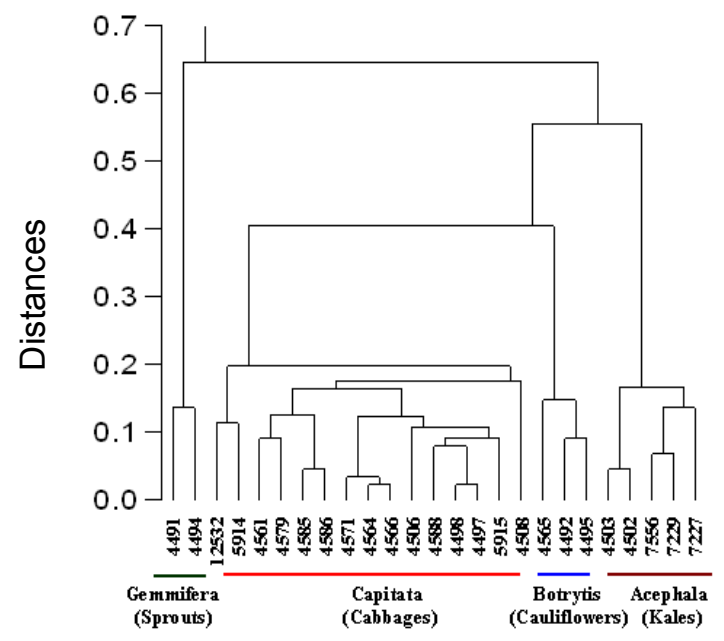

Figure 3. Dendrogram showing the relationship among 25 accessions of Brassica oleracea based on morphological data

\subsection{Jaccard Binary Similarity Coefficients (Sj)}

The overall mean similarity index for Brassica oleracea accessions calculated by the Jaccard similarity index ( $\mathrm{Sj}$ ) ranged from 0.051 to 0.976 with an average of 0.503 (Table 4). The highest similarity indices $(0.976)$ were between the accessions of the same crop variety and geographical region, e.g. cabbage HRIGRU4497 and HRIGRU4498 from Roscommon. The second indices (0.952) were also between the accessions of the same group and geographical region, e.g. spring cabbages HRIGRU4566 and HRIGRU4564 from Cork, and spring cabbages HRIGRU4564 and HRIGRU4571 from Cork. The Sj similarity between the accessions of fodder kales HRIGRU4502 and HRIGRU4503 from Kildare was about 0.93 . The accession of common cabbage HRIGRU4585 from Donegal had a high similarity index (0.907) with the accession of common cabbage HRIGRU4586 from Mayo. The Sj similarity between the accessions of common cabbage HRIGRU4586 and cabbage HRIGRU5915 was about 0.64 . The lowest similarity indices and the highest variation were found between the accessions of kale HRIGRU7227 from Sligo and brussels sprout HRIGRU4494 from Dublin. The highest genetic distance in the cabbage types was found between the accessions of cabbage HRIGRU12532 and cattle cabbages HRIGRU4579, HRIGRU4561 and HRIGRU4508. 
Table 4. Sj Jaccard binary similarity coefficients calculated from the morphological data for 25 accessions of

Brassica oleracea (see Table 1 for identification of accessions numbers)

\begin{tabular}{|c|c|c|c|c|c|c|c|c|c|c|c|c|c|c|c|c|c|c|c|c|c|c|c|c|c|}
\hline & 1 & 2 & 3 & 4 & 5 & 6 & 7 & 8 & 9 & 10 & 11 & 12 & 13 & 14 & 15 & 16 & 17 & 18 & 19 & 20 & 21 & 22 & 23 & 24 & 25 \\
\hline 1 & 1 & & & & & & & & & & & & & & & & & & & & & & & & \\
\hline 2 & 0.930 & 1 & & & & & & & & & & & & & & & & & & & & & & & \\
\hline 3 & 0.750 & 0.729 & 1 & & & & & & & & & & & & & & & & & & & & & & \\
\hline 4 & 0.750 & 0.729 & 0.867 & 1 & & & & & & & & & & & & & & & & & & & & & \\
\hline 5 & 0.660 & 0.640 & 0.804 & 0.729 & 1 & & & & & & & & & & & & & & & & & & & & \\
\hline 6 & 0.277 & 0281 & 0317 & 0339 & 0.281 & 1 & & & & & & & & & & & & & & & & & & & \\
\hline 7 & 0.277 & 0281 & 0.277 & 0297 & 0.242 & 0.745 & 1 & & & & & & & & & & & & & & & & & & \\
\hline 8 & 0.277 & 0281 & 0317 & 0339 & 0.281 & 0.822 & 0.708 & 1 & & & & & & & & & & & & & & & & & \\
\hline 9 & 0.292 & 0277 & 0.292 & 0.292 & 0.221 & 0.407 & 0.383 & 0.383 & 1 & & & & & & & & & & & & & & & & \\
\hline 10 & 0.239 & 0242 & 0.239 & 0.239 & 0.206 & 0.414 & 0.414 & 0390 & 0.844 & 1 & & & & & & & & & & & & & & & \\
\hline 11 & 0.258 & 0.242 & 0.297 & 0.258 & 0.242 & 0.390 & 0.390 & 0.367 & 0.766 & 0.745 & 1 & & & & & & & & & & & & & & \\
\hline 12 & 0.258 & 0262 & 0.258 & 0.258 & 0.224 & 0.464 & 0.439 & 0.439 & 0.766 & 0.783 & 0.783 & 1 & & & & & & & & & & & & & \\
\hline 13 & 0.239 & 0242 & 0.258 & 0.258 & 0.206 & 0.390 & 0.344 & 0367 & 0.804 & 0.745 & 0.673 & 0.745 & 1 & & & & & & & & & & & & \\
\hline 14 & 0.239 & 0242 & 0.258 & 0.258 & 0.206 & 0.390 & 0.344 & 0.367 & 0.804 & 0.745 & 0.673 & 0.745 & 0.907 & 1 & & & & & & & & & & & \\
\hline 15 & 0.254 & 0239 & 0.292 & 0.292 & 0.239 & 0.456 & 0.383 & 0.431 & 0.714 & 0.694 & 0.729 & 0.804 & 0.660 & 0.660 & 1 & & & & & & & & & & \\
\hline 16 & 0.239 & 0242 & 0.277 & 0.277 & 0.242 & 0.464 & 0.390 & 0.439 & 0.694 & 0.708 & 0.708 & 0.822 & 0.673 & 0.673 & 0.976 & 1 & & & & & & & & & \\
\hline 17 & 0.258 & 0262 & 0.297 & 0.297 & 0.242 & 0.439 & 0.367 & 0.414 & 0.729 & 0.673 & 0.673 & 0.783 & 0.708 & 0.708 & 0.844 & 0.864 & 1 & & & & & & & & \\
\hline 18 & 0.239 & 0224 & 0317 & 0.277 & 0262 & 0.439 & 0.367 & 0.414 & 0.694 & 0.673 & 0.783 & 0.822 & 0.673 & 0.640 & 0.886 & 0.864 & 0.822 & 1 & & & & & & & \\
\hline 19 & 0.258 & 0262 & 0.258 & 0258 & 0.242 & 0.390 & 0.367 & 0367 & 0.596 & 0.577 & 0.577 & 0.673 & 0.608 & 0.608 & 0.660 & 0.673 & 0.745 & 0.640 & 1 & & & & & & \\
\hline 20 & 0.258 & 0262 & 0.258 & 0.258 & 0.242 & 0.414 & 0.390 & 0.390 & 0.729 & 0.708 & 0.673 & 0.783 & 0.673 & 0.673 & 0.766 & 0.783 & 0.783 & 0.745 & 0.708 & 1 & & & & & \\
\hline 21 & 0.277 & 0281 & 0.277 & 0277 & 0.224 & 0.414 & 0.390 & 0.390 & 0.766 & 0.708 & 0.673 & 0.783 & 0.708 & 0.708 & 0.766 & 0.783 & 0.822 & 0.745 & 0.673 & 0.952 & 1 & & & & \\
\hline 22 & 0.277 & 0281 & 0.277 & 0.277 & 0.224 & 0.414 & 0.390 & 0390 & 0.804 & 0.745 & 0.673 & 0.783 & 0.708 & 0.745 & 0.766 & 0.783 & 0.822 & 0.745 & 0.673 & 0.907 & 0.952 & 1 & & & \\
\hline 23 & 0.258 & 0262 & 0.297 & 0.297 & 0.281 & 0.439 & 0.367 & 0.414 & 0.596 & 0.577 & 0.547 & 0.708 & 0.673 & 0.673 & 0.694 & 0.708 & 0.708 & 0.708 & 0.783 & 0.745 & 0.708 & 0.708 & 1 & & \\
\hline 24 & 0.091 & 0.078 & 0.091 & 0.120 & 0.064 & 0.203 & 0.221 & 0.221 & 0.273 & 0.277 & 0277 & 0.258 & 0.239 & 0.239 & 0.273 & 0.258 & 0239 & 0.258 & 0.169 & 0221 & 0.221 & 0.221 & 0.186 & 1 & \\
\hline 25 & 0.064 & 0.065 & 0.064 & 0.092 & 0.051 & 0.188 & 0.206 & 0.206 & 0.239 & 0.262 & 0.242 & 0.242 & 0.224 & 0.224 & 0.239 & 0.242 & 0.224 & 0224 & 0.155 & 0.206 & 0.206 & 0.206 & 0.171 & 0.766 & 1 \\
\hline
\end{tabular}

\subsection{Principal Component Analysis (PCA)}

Principal component analysis (PCA) showed variations among the accessions studied (Table 5). The first 5 varieties of the principal component analysis accounted for $87.24 \%$ of the total variation among the 25 accessions. The first component (PC1) explained $37.88 \%$ of the total variation, while PC2, PC3, PC4 and PC5 contributed $27.97 \%, 11.89 \%, 5.13 \%$ and $4.38 \%$ of the total variation, respectively.

Table 5. Matrix of eigenvectors and values of the principal components for the morphological traits of 25 accessions of Brassica oleracea

\begin{tabular}{lccccc}
\hline & \multicolumn{5}{c}{ Principal components } \\
& PC1 & PC2 & PC3 & PC4 & PC5 \\
\hline Variance Explained by Components & 15.908 & 11.747 & 4.993 & 2.156 & 1.839 \\
Percent of Total Variance Explained & 37.88 & 27.97 & 11.89 & 5.13 & 4.38 \\
Accumulated Eigenvectors & 37.875 & 65.844 & 77.733 & 82.866 & 87.244 \\
Seedling leaf colour & 0.102 & 0.080 & -0.078 & 0.416 & 0.305 \\
Seedling pubesence & -0.162 & -0.341 & -0.344 & 0.695 & -0.115 \\
Juvenile development & -0.264 & -0.177 & -0.255 & -0.123 & 0.701 \\
Plant growth habit & -0.091 & 0.478 & 0.821 & -0.038 & -0.185 \\
Lodging & 0.450 & -0.668 & 0.161 & 0.143 & 0.468 \\
No. of leaves (estimated) & 0.591 & 0.082 & 0.062 & 0.220 & 0.644 \\
Leaf blade shape in outline & -0.212 & -0.016 & 0.959 & 0.136 & 0.067 \\
Leaf division (margin) & 0.017 & -0.984 & 0.155 & -0.027 & -0.012 \\
Leaf division (incision) & 0.306 & -0.159 & -0.157 & 0.740 & -0.207 \\
Leaf apex shape & 0.708 & 0.200 & -0.438 & -0.367 & 0.098 \\
Leaf colour & 0.115 & 0.549 & 0.121 & -0.165 & 0.000 \\
Leaf hairiness & -0.399 & -0.097 & -0.170 & 0.370 & 0.541 \\
Leaf bloom & 0.017 & -0.984 & 0.155 & -0.027 & -0.012 \\
Petiole section & -0.142 & 0.405 & 0.862 & 0.046 & 0.131 \\
Petiole and/or midvein colour & 0.092 & 0.518 & -0.154 & 0.446 & -0.242
\end{tabular}




\begin{tabular}{lccccc} 
Heading habit & 0.954 & 0.193 & 0.206 & 0.022 & -0.003 \\
Head shape in longitudinal section & 0.954 & 0.193 & 0.206 & 0.022 & -0.003 \\
Head-forming leaf overlap at terminal region & 0.972 & 0.181 & -0.116 & 0.006 & -0.039 \\
Head cover from subtending leaves & 0.996 & -0.007 & 0.007 & 0.006 & -0.032 \\
Primary colour of outer head leaves & 0.866 & 0.351 & 0.204 & -0.064 & 0.075 \\
Head size in relation to plant size & 0.976 & -0.199 & 0.037 & 0.000 & -0.033 \\
Head solidity & 0.954 & 0.193 & 0.206 & 0.022 & -0.033 \\
Head length & 0.945 & -0.301 & -0.020 & -0.007 & -0.015 \\
Head diameter & 0.938 & -0.317 & -0.028 & 0.010 & -0.038 \\
Stem length in head, estimate & 0.954 & 0.193 & 0.206 & 0.022 & -0.003 \\
Primary colour inside cut & 0.631 & 0.473 & -0.131 & -0.050 & 0.106 \\
Tendency of head to split & 0.796 & 0.360 & 0.349 & -0.032 & 0.088 \\
Head holding ability (bolting resistance) & 0.954 & 0.193 & 0.206 & 0.022 & -0.003 \\
Relative time of maturity & -0.841 & -0.137 & 0.511 & 0.015 & 0.078 \\
Leaf retention on stem & 0.154 & -0.911 & 0.208 & -0.154 & -0.198 \\
Number of shoots or enlarged buds & -0.017 & 0.984 & -0.155 & 0.027 & 0.012 \\
Size of enlarged vegetative buds & -0.017 & 0.984 & -0.155 & 0.027 & 0.012 \\
Firmness of enlarged vegetative buds & -0.017 & 0.984 & -0.155 & 0.027 & 0.012 \\
Colour of enlarged vegetative buds or shoots & -0.017 & 0.959 & -0.154 & 0.004 & 0.022 \\
Floral apex branching pattern & -0.034 & 0.042 & 0.988 & 0.048 & 0.108 \\
Days to flowering & -0.949 & -0.020 & 0.268 & 0.029 & -0.024 \\
Flower stalk colour & -0.959 & 0.144 & -0.127 & 0.001 & 0.025 \\
Flower stalk length, estimate & -0.627 & 0.735 & 0.241 & 0.034 & 0.067 \\
Flower stalk internode length & -0.627 & 0.735 & 0.241 & 0.034 & 0.067 \\
Flowering plant hairiness & -0.017 & 0.984 & -0.155 & 0.027 & 0.012 \\
Flower colour variability in the crop & -0.008 & 0.699 & -0.100 & 0.097 & -0.029 \\
Petal colour & -0.256 & 0.070 & -0.286 & -0.544 & 0.222 \\
\hline & & & & & \\
\hline
\end{tabular}

The values of the principal component analysis for all morphological traits were shown in Table 5 . The traits, which showed the greatest variability on the first component, were leaf apex shape (0.708), heading habit (0.954), head shape in longitudinal section (0.954), head-forming leaf overlap at terminal region (0.972), head cover from subtending leaves $(0.996)$, primary colour of outer head leaves $(0.866)$, head size in relation to plant size $(0.976)$, head solidity (0.954), head length (0.945), head diameter (0.938), stem length in head estimated (0.954), tendency of head to split (0.796) and head holding ability (0.954).

Leaf colour (0.549), petiole and/or midvein colour (0.518), number of shoots or enlarged buds (0.984), size of enlarged vegetative buds (0.984), firmness of enlarged vegetative buds (0.984), colour of enlarged vegetative buds or shoots (0.959), flower stalk length estimated (0.735), flower stalk internode length $(0.735)$, flowering plant hairiness $(0.984)$ and flower colour variability in the crop (0.699) were highly and positively correlated with the second component.

The traits which showed the greatest influence in the third component, were plant growth habit (0.821), leaf blade shape in outline (0.959), leaf margin (0.155), leaf bloom (0.155), petiole section $(0.862)$, relative time of maturity (0.511), leaf retention on stem (0.208), floral apex branching pattern $(0.988)$ and days to flowering $(0.268)$. The fourth component separated three traits; seedling leaf colour $(0.416)$, seedling pubesence $(0.695)$ and leaf incision (0.740). Juvenile development (0.701), lodging (0.468), number of leaves estimated $(0.644)$, leaf hairiness $(0.541)$ and petal colour $(0.222)$ showed the greatest variability on the fifth component. The values of the principal components of flower stalk colour, leaf margin, leaf bloom, leaf retention on stem traits were low (Table 5). However, these traits exhibited some degree of variation among the accessions of Brassica oleracea.

\subsection{Analysis of Variance (ANOVA)}

Of the 44 morphological traits examined in this study, 28 were significantly different among the accessions of Brassica oleracea (Table 6). These 28 significant descriptors were deemed useful and can be used for further characterisation of the Brassica accessions. The regression coefficients $\left(\mathrm{r}^{2}\right)$ values ranged from 0.041 to 0.55 with an average of 0.31 . 
Table 6. One-way ANOVA for the morphological traits among 25 accessions of Brassica oleracea

\begin{tabular}{|c|c|c|}
\hline Dependent variable (traits) & $\begin{array}{c}\mathbf{r}^{2} \text { (regression } \\
\text { coefficients) }\end{array}$ & Probability (P) \\
\hline Seedling leaf colour & 0.041 & 0.026 \\
\hline Seedling pubesence & 0.088 & 0.179 \\
\hline Juvenile development & 0.074 & $0.001 *$ \\
\hline Plant growth habit & 0.154 & $0.000 * *$ \\
\hline Lodging & 0.234 & $0.001^{*}$ \\
\hline No. of leaves (estimated) & 0.164 & $0.000 * *$ \\
\hline Leaf blade shape in outline & 0.059 & 0.011 \\
\hline Leaf division (margin) & 0.309 & $0.000 * *$ \\
\hline Leaf division (incision) & 0.082 & $0.001 *$ \\
\hline Leaf apex shape & 0.271 & 0.317 \\
\hline Leaf colour & 0.118 & $0.000 * *$ \\
\hline Leaf hairiness & 0.107 & $0.000 * *$ \\
\hline Leaf bloom & 0.317 & $0.000 * *$ \\
\hline Petiole section & 0.133 & $0.000 * *$ \\
\hline Petiole and/or midvein colour & 0.120 & $0.000 * *$ \\
\hline Heading habit & 0.509 & $0.003 *$ \\
\hline Head shape in longitudinal section & 0.490 & $0.003 *$ \\
\hline Head-forming leaf overlap at terminal region & 0.484 & $0.001 *$ \\
\hline Head cover from subtending leaves & 0.483 & 0.018 \\
\hline Primary colour of outer head leaves & 0.388 & $0.000 * *$ \\
\hline Head size in relation to plant size & 0.459 & $0.001^{*}$ \\
\hline Head solidity & 0.392 & $0.003^{*}$ \\
\hline Head length & 0.421 & 0.703 \\
\hline Head diameter & 0.389 & 0.443 \\
\hline Stem length in head, estimate & 0.324 & $0.003^{*}$ \\
\hline Primary colour inside cut & 0.253 & 0.125 \\
\hline Tendency of head to split & 0.230 & 0.326 \\
\hline Head holding ability (bolting resistance) & 0.244 & $0.003 *$ \\
\hline Relative time of maturity & 0.253 & 0.072 \\
\hline Leaf retention on stem & 0.536 & $0.000 * *$ \\
\hline Number of shoots or enlarged buds & 0.540 & $0.000 * *$ \\
\hline Size of enlarged vegetative buds & 0.498 & $0.000 * *$ \\
\hline Firmness of enlarged vegetative buds & 0.448 & $0.000 * *$ \\
\hline Colour of enlarged vegetative buds or shoots & 0.362 & $0.000 * *$ \\
\hline Floral apex branching pattern & 0.170 & $0.000 * *$ \\
\hline Days to flowering & 0.367 & 0.064 \\
\hline Flower stalk colour & 0.343 & $0.001 *$ \\
\hline Flower stalk length, estimate & 0.550 & 0.006 \\
\hline Flower stalk internode length & 0.437 & 0.006 \\
\hline Flowering plant hairiness & 0.497 & $0.000 * *$ \\
\hline Flower colour variability in the crop & 0.510 & $0.000 * *$ \\
\hline Petal colour & 0.065 & 0.072 \\
\hline
\end{tabular}

* and ** are significantly different at the $\mathrm{P}<0.005$ and $\mathrm{P}<0.001$ respectively. 


\subsection{Correlation Coefficients among the Morphological Traits}

Pearson correlation coefficients were computed for all pairs of traits (data not shown). The highest correlation was between leaf margin and leaf bloom $(\mathrm{R}=1)$, and flower stalk length estimated and flower stalk internode length $(\mathrm{R}=1)$. There was a high correlation between flowering plant hairiness trait and some of enlarged buds traits, e.g. firmness, size and number of enlarged buds $(\mathrm{R}=1)$. There was also a high correlation among head traits (heading habit, head shape in longitudinal section, head solidity, stem length in head and head holding ability) $(\mathrm{R}=1)$. The head holding ability (bolting resistance) was high in most of the accessions studied. On the contrary, leaf margin and leaf bloom were negatively correlated with flowering plant hairiness and most enlarged buds traits, e.g. number, size and firmness $(\mathrm{R}=-1)$. Days to flowering trait was also negatively correlated to most traits, e.g. number of leaves estimated, leaf apex shape, leaf retention on stem, stem length in head, head length, size and number of enlarged buds, and all head and enlarged buds traits.

\section{Discussion}

To conserve and utilise germplasm efficiently, it is essential to investigate the extent of diversity available. Morphological characterisation is an essential step in the characterisation and classification of crop germplasm because a breeding program mainly relies on the magnitude of morpho-phenological variability (Koffi et al., 2008). The morphological traits used in this study showed a pronounced variation among accessions. Seedling traits such as juvenile development and seedling leaf colour showed the lowest variation. The hypocotyl colour and seedling leaf (marginal incisions) traits had no taxonomic value among accessions of Brassica oleracea because these traits showed the same patterns within all of the accessions studied. However, they could be used to assess the variation among wild and cultivated species of genus Brassica. Vegetative preflowering and maturity traits such as petiole section, petiole and midvein colour, leaf colour, primary colour of outer head leaves, leaf division (incision), etc. exhibited a high level of variation among the accessions studied. These results were consistent with that reported by Rabbani et al. (1999).

The cluster analysis showed that the cauliflowers were more closely related to the cabbages than the kales were. However, the kales were more closely related to cauliflowers and cabbages than the brussels sprouts were. The accessions of most cabbage types were distributed among different clusters within cabbage subgroups, and this agreed with Balkaya et al. (2005), who reported that there was some degree of variation among Turkish white head cabbages. However, the cluster analysis from this current study is more reliable, as most of the accessions of each group and subgroup were collected from closely related geographical regions. Only a few accessions were scattered among the different subgroups. This showed a sort of correlation between clustering pattern and eco-geographical distribution of the accessions. This result agreed with that reported by Faltusová et al. (2011), but was not consistent with that reported by Balkaya et al. (2005). The difference in these data could be attributed to the differences in accessions or the traits used which could in turn be influenced by environmental factors such as geographical area, elevation of temperature, and soil fertility.

The output of the Jaccard binary similarity coefficient from our morphological data revealed that the highest similarity indices were between the accessions of the same crop variety and geographical region. This confirmed the correlation between grouping on morphological data and eco-geographical distribution of the accessions. However, this high similarity could be attributed to the low gene flow within the same crop variety. This study also suggested that the accessions of kale HRIGRU7227, common cabbage HRIGRU4586, spring cabbage HRIGRU5914, cattle cabbage HRIGRU4561, HRIGRU4508 and HRIGRU4579 and cabbage HRIGRU12532, HRIGRU5915 and HRIGRU4588, due to the highest variation found among them, could provide the opportunities for selecting as parental source in future breeding programs to develop new or more productive Brassica varieties.

In the analysis made to estimate the relative contributions of the different morphological traits studied towards the overall phenotypic variation among the 25 accessions, a total of 5 principal components (PCs) explained 87.24\% of the total variation in all the 44 traits studied. This data indicated a high degree of correlation among traits of the accessions studied. About $38 \%$ of the total variability explained by the first PC alone was due chiefly to variations in enlarged apical buds (head) traits, indicating a very high degree of correlation among them. Furthermore, all enlarged vegetative buds and most of enlarged inflorescences (floral branching pattern) traits were separated on the second component (explained about $28 \%$ of the total variability), indicating a high degree of correlation among them. We could thus conclude that the traits of enlarged apical buds, enlarged vegetative buds and enlarged inflorescences proved to be useful markers for separating Brassica oleracea varieties. These observations agreed with the classification criteria of Brassica oleracea varieties reported by Paterson et al. (2001) and Hong et al. (2008), that are mainly based on extreme morphological characteristics such as the enlarged single apical bud of 
cabbage, the several enlarged vegetative buds of brussels sprout and the enlarged inflorescences (floral branching pattern) of cauliflower.

One-way analysis of variance (ANOVA) of the morphological traits used showed that 28 traits were significantly different among the accessions studied, and can be used for further characterisation of Brassica accessions. However, the low percent of contribution of the morphological traits (31\%) could be attributed to the low number of flowering traits used. Furthermore, most of accessions were of orbicular leaf blade shape, dentate leaf margin, rounded leaf apex, green leaf colour, intermediate leaf bloom, rounded petiole section, spheric head shape, strong head forming leaf overlap, covered head, green primary colour of outer head leaves, yellow green flower stalk, short flower stalk length and pale yellow petals. These results were relatively consistent with that reported by Katz (2003).

The correlation is a measure of the degree to which variables differ or a measure of the intensity of association (Rabbani et al., 1999). The correlations revealed by Pearson correlation matrix in this study suggest that when the flower stalk length estimated is used as a distinguishing character, the flower stalk internode length could be redundant for characterizing the accessions. Most of the highly correlated traits (e.g. heading habit, head solidity, stem length in head firmness, size and number of enlarged buds and flower stalk length estimated) are of economic importance and are identified as useful for Brassica breeding programs and taxonomic purposes.

\section{Conclusion}

This study differentiated all the accessions of Brassica oleracea studied, and identified 28 morphological traits which were significantly different among the accessions and can be used for further characterisation of Brassica accessions. It also identified 9 highly diverse accessions, providing opportunities for optimising parental sources in future breeding programmes to develop new or more productive Brassica varieties. Morphological traits proved useful in assessing the diversity and relationships of Irish Brassica oleracea germplasm.

\section{Acknowledgements}

We would like to thank Dr. Charlotte Allender (University of Warwick, Wellsbourne, United Kingdom) for providing us with the plant material. We also wish to thank Dr. Barry Murphy (Teagasc Horticultural Research centre, Kinsealy, Ireland) and Prof. Dr. Reda Sammour (Tanta University, Egypt) for their help and fruitful discussions during this research. This work was financially supported by the Department of Agriculture, Fisheries and Food (DAFF) under the Conservation of Genetic Resources for Food and Agriculture Scheme 2009 and the Dublin Institute of Technology ABBEST Scholarship Scheme, Ireland.

\section{References}

Arshad, M., Aslam, M., \& Irshad, M. (2009). Genetic variability and character association among morphological traits of mungbean, Vigna radiata L. Wilczek genotypes. J. Agric. Res., 47, 121-126.

Balkaya, A., Yanmaz, R., \& Kar, A. A. (2005). Morphological characterisation of white head cabbage (Brassica oleracea var. capitata subvar. alba) genotypes in Turkey. N. Z. J. Crp. Hort. Sci., 33, 333-341.

Bibi, S., Khan, I., Bughio, H., Odhano, I., Asad, M. A., \& Khatri, A. (2009). Genetic differentiation of rice mutants based on morphological traits and molecular marker (RAPD). Pak. J. Bot., 41, 737-743.

Christopher, G. L., Andrew, J. R., Geraldine, A. C. L., Clare, J. H., Jacqueline, B., Gary, B., German, C. S., \& David, E. (2005). Brassica ASTRA: an integrated database for Brassica genomic research. Nucleic Acids Res., 33, D656-D659. http://dx.doi.org/10.1093/nar/gki036

Hong, C. P., Kwon, S. J., Kim, J. S., Yang, T. J., Park, B. S., \& Lim, Y. P. (2008). Progress in Understanding and Sequencing the Genome of Brassica rapa. Int. J. Plant Genomics, pp. 1-9 http://dx.doi.org/10.1155/2008/582837

IBPGR. (1990). Descriptors for Brassica and Raphanus. International Board for Plant Genetic Resources, Rome, Italy.

Iqbal, Z., Arshad, M., Ashraf, M., Mahmood, T., \& Waheed, A. (2008). Evaluation of soybean [Glycine max (L.) Merrill] germplasm for some important morphological traits using multivariate analysis. Pak. J. Bot., 40(6), 2323-2328.

Karp, A., Kresovich, S., Bhat, K. V., Ayad, W. G., \& Hodgkin, T. (1997). Molecular tools in plant genetic resources conservation: a guide to the technologies. International Plant genetic Resources Institute (IPGRI). IPGRI Technical Bulletin No. 2. 
Katz, S. H. (2003). Cabbage and Crucifer plants. Encyclopedia of Food \& Culture, vol. 1. Gale Cengage. eNotes.com. 2006. Available online at http://www.enotes.com/food-encyclopedia/cabbage-crucifer-plants. accessed 5 August 2010.

King, G. J. (2005). A white paper for the multinational Brassica genome project. Available online at http://www.brassica.info/info/publications/white_paper.php

Koffi, K. K., Gbotto, A. A., Malice, M., Djè, Y., Bertin, P., Baudoin, J., \& Zoro Bi, I. A. (2008). Morphological and allozyme variation in a collection of Cucumeropsis mannii Naudin (Cucurbitaceae) from Co te d'Ivoire. Biochem. Sys. Ecol., 36, 777-789. http://dx.doi.org/10.1016/j.bse.2008.07.008

Kop, E. P., Teakle, G. R., McClenaghan, E. R., Lynn J. R., \& King G. J. (2003). Genetic analysis of the bracting trait in cauliflower and broccoli. Plant Science, 164:803-808. http://dx.doi.org/10.1016/S0168-9452(03)00068-2

Legendre, P., \& Legendre, L. (1998). Numerical Ecology. Developments in Environmental Modelling, 20. Elsevier, Amsterdam.

National Biodiversity Plan (NBP). (2011). Government of Ireland, ACTIONS FOR BIODIVERSITY 2011-2016. Available online at http://www.cbd.int/doc/world/ie/ie-nbsap-v2-en.pdf

Paterson, A. H., Lan, T. H., Amasino, R., Osborn, T. C., \& Quiros, C. (2001). Brassica genomics: a complement to, and early beneficiary of, the Arabidopsis sequence. Genome Biology, 2(3), 1011.1-1011.4. http://dx.doi.org/10.1186/gb-2001-2-3-reviews1011

Rabbani, M. A., Iwabuchi, A., Murakami, Y., Suzuki, T., \& Takayanagi, K. (1999). Collection, Evaluation and Utilization of Oilseed Mustard (Brassica juncea L.) in Pakistan. Pak. J. Biol. Sci., 2(1), 88-94. http://dx.doi.org/10.3923/pjbs.1999.88.94

Rakow, G. (2004). Species origin and economic importance of Brassica. In: Pua EC, Douglas CJ, editors. Biotechnology in Agriculture and Forestry. Vol. 54. New York: Springer-Verlag Berlin Heidelberg, pp. 3-11.

Rich, T. C. G. (1991). Crucifers of Great Britain and Ireland. Botanical Society of the British Isles (BSBI), London, pp. 336.

Rohlf, F. (2000). Ntsyspc, numerical taxonomy and multivariate analysis system. New York: Exeter Pub., Ltd

Rohlf, F., Chang, W., Sokal, R., \& Kim, J. (1990). Accuracy of estimated phylogenies: Effects of tree topology and evolutionary model. Evolution, 44, 1671-1684.

Saha, S., Molla, M.R., Chandra, D., \& Rahman, L. (2008). Assessment of genetic variation and relationships within the varieties of four Brassica species by RAPD markers. Aust. J. Crop Sci., 2(3), 105-114.

Tantasawat, P., Trongchuen, J., Prajongjai, T., Thongpae, T., Petkhum, C., Seehalak, W., \& Machikowa, T. (2010). Variety identification and genetic relationships of mungbean and blackgram in Thailand based on morphological characters and ISSR analysis. Afr. J. Biotechnol, 9(27), 4452-4464.

Tümbilen, Y., Frary, A., Mutlu, S., \& Doğanlar, S. (2011). Genetic diversity in Turkish eggplant (Solanum melongena) varieties as determined by morphological and molecular analyses. International Research Journal of Biotechnology (IRJOB), 2(1), 16-25.

Zhao, J. (2007). The genetics of phytate content and morphological traits in Brassica rapa. PhD thesis, Wageningen University, The Netherlands. 